\title{
Expression of a Functional Secreted YopN-TyeA Hybrid Protein in Yersinia pestis Is the Result of a +1 Translational Frameshift Event
}

\author{
Franco Ferracci, $\uparrow$ James B. Day, $\uparrow+$ Heather J. Ezelle, $\dagger$ and Gregory V. Plano* \\ Department of Microbiology and Immunology, University of Miami School of Medicine, Miami, Florida 33101
}

Received 20 February 2004/Accepted 21 April 2004

\begin{abstract}
YopN is a secreted protein that prior to secretion directly interacts with the cytosolic SycN/YscB chaperone complex and TyeA. This study identifies a secreted YopN-TyeA hybrid protein that is expressed by Yersinia pestis, but not by Yersinia enterocolitica. DNA sequence analysis and site-directed mutagenesis studies demonstrate that the hybrid protein is the result of a +1 translational frameshift event.
\end{abstract}

Yersinia pestis is the causative agent of plague, an invasive and often fatal disease of animals and humans. The pathogenicity of $Y$. pestis primarily results from its ability to avoid the defenses of its mammalian host while overwhelming it with massive growth (6). These capabilities are strictly dependent upon the presence of a 70-kb plasmid termed pCD1 in $Y$. pestis KIM (24). Plasmid pCD1 and related plasmids (2) in the other human pathogenic yersiniae ( $Y$. enterocolitica and $Y$. pseudotuberculosis) encode a set of secreted antihost proteins termed Yersinia outer proteins (Yops) and a unique delivery system, classified as a type III secretion (TTS) system $(2,16)$. This system allows extracellular yersiniae to inject Yops directly into host phagocytic cells $(15,25,27)$. Injected Yops function to prevent bacterial phagocytosis and block production of proinflammatory cytokines $(22,23,30)$.

Yop secretion is triggered by contact between a bacterium and the surface of a targeted host cell. Following cell contact, effector Yops are injected into the eukaryotic cell and are not found in substantial amounts in the extracellular milieu, indicating that the delivery process is polarized $(2,28)$. In vitro, Yop secretion is blocked in the presence of millimolar levels of extracellular calcium and triggered during growth at $37^{\circ} \mathrm{C}$ in the absence of calcium $(21,33)$. The secreted YopN protein $(13,35)$ and the cytosolic TyeA $(5,18), \operatorname{SycN}(8,17)$, YscB $(19)$, and $\operatorname{LcrG}(20,29)$ proteins are required to prevent Yop secretion in the presence of calcium and prior to contact with a eukaryotic cell. Mutational inactivation of any one of the five genes encoding these proteins results in uncontrolled secretion prior to host cell contact and a loss of polarized injection following cell contact.

SycN and YscB interact with one another to form a SycN/ YscB complex that functions as a TTS chaperone for YopN (8). The SycN/YscB complex binds to an N-terminal region of YopN and is required for efficient YopN secretion (Fig. 1).

\footnotetext{
* Corresponding author. Mailing address: Department of Microbiology and Immunology, University of Miami School of Medicine, P.O. Box 016960 (R-138), Miami, FL 33101. Phone: (305) 243-6310. Fax: (305) 243-4623. E-mail: gplano@med.miami.edu.

$\dagger$ F.F., J.B.D., and H.E. contributed equally to this work.

$\$$ Present address: Center for Food Safety and Applied Nutrition, Food and Drug Administration, College Park, MD 20740.
}

Tye A is a cytosolic protein that binds to a C-terminal region of YopN and inhibits YopN secretion $(4,7,18)$. The mechanism by which the YopN-SycN-YscB-TyeA complex regulates Yop secretion is unknown; however, it has been hypothesized that partially secreted YopN bound by TyeA directly blocks the secretion channel prior to contact with a eukaryotic cell (4). Upon contact with a eukaryotic cell, an as-yet-unidentified signaling event allows YopN to complete its secretion, thus clearing the secretion channel for delivery of the effector Yops. Secreted YopN is directed into the eukaryotic cell (7); however, no direct antihost function has been attributed to this protein.

Identification of a secreted YopN-TyeA hybrid protein in $\boldsymbol{Y}$. pestis. Sodium dodecyl sulfate-polyacrylamide gel electrophoresis (SDS-PAGE) and immunoblot analysis of cell pellet and culture supernatant fractions from $Y$. pestis KIM8-3002 (34), Y. pseudotuberculosis YPIII (3), and Y. enterocolitica 8081V (26) with antiserum specific for YopN identified a ca. 42-kDa secreted protein in the $Y$. pestis and $Y$. pseudotuberculosis strains that was absent from $Y$. enterocolitica (Fig. 2A; $Y$. pseudotuberculosis data not shown). The novel secreted protein was significantly larger than the ca. $32-\mathrm{kDa}$ secreted YopN protein observed in all three strains. A secreted protein of ca. $42 \mathrm{kDa}$ was also identified in $Y$. pestis cell pellet and culture supernatant fractions probed with antiserum specific for TyeA (Fig. 2C). This was noteworthy because previous reports had shown that TyeA is a ca. $10-\mathrm{kDa}$ cytosolic protein (5) that interacts with cytosolic YopN. The size of the anti-YopN $(\alpha-$ YopN)- and $\alpha$-TyeA-reactive band was approximately the size of a YopN-TyeA heterodimer.

The predicted amino acid sequences of YopN and TyeA each contained a single cysteine residue (YopN Cys140 and TyeA Cys73), indicating that a YopN-TyeA disulfide-linked heterodimer was possible. The contribution of disulfide bonds to the formation of the $42-\mathrm{kDa}$ band was evaluated by analyzing the samples under reducing (5\% $\quad \beta$-mercaptoethanol $[\beta \mathrm{ME}])$ and nonreducing $(-\beta \mathrm{ME})$ conditions. No change in the size or amount of the $42-\mathrm{kDa}$ product in the presence or absence of $5 \% \beta \mathrm{ME}$ was observed (data not shown), indicating that the $42-\mathrm{kDa}$ band was not a disulfide-linked YopN-TyeA heterodimer.

To assess the role of YopN and TyeA in the formation of the 

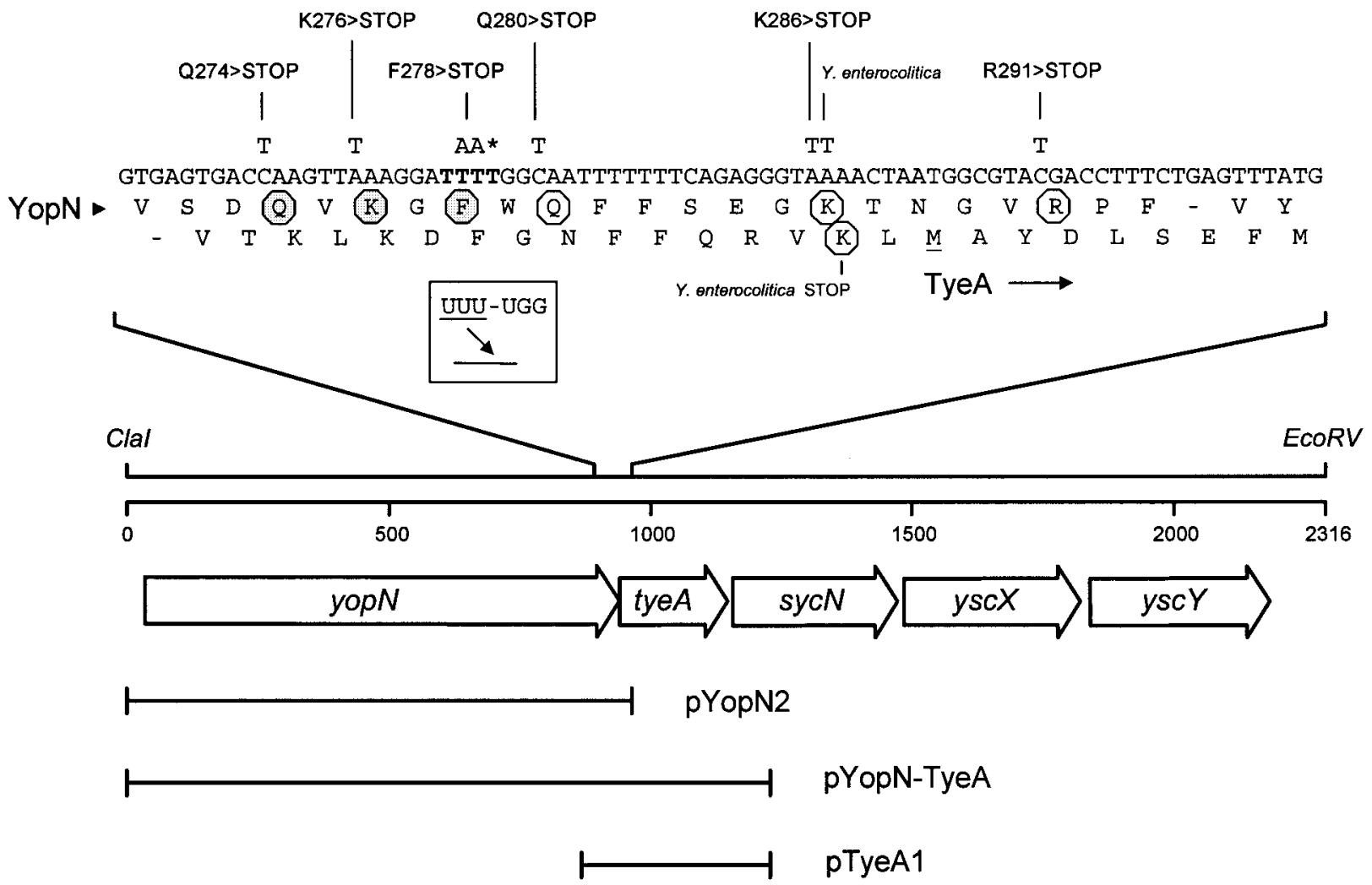

FIG. 1. Genetic map of the $Y$. pestis yopNtyeAsycNysc $X Y$ operon. The DNA sequences of the $3^{\prime}$ region of yopN and the $5^{\prime}$ region of tye $A$ are shown. The location of the putative +1 translational frameshift site is shown in boldface type and illustrated in the inset box. The location of the Q274>STOP, K276>STOP, F278>STOP, Q280>STOP, K286>STOP, and R291>STOP changes used to map the site of the translational frameshift are indicated (octagons). Termination codons (octagons) that eliminate production of the YopN-TyeA hybrid protein are shaded. The location of the $\mathrm{T}$ in $Y$. enterocolitica that inserts a stop codon immediately upstream of the TyeA start codon is also indicated (octagon). The location of the single $\mathrm{T}$ residue deleted in plasmid pYopN-TyeA-FS1 is indicated by an asterisk. DNA fragments used in the construction of plasmids pYopN2, pYopN-TyeA, and pTyeA1 are shown.

42-kDa band, $Y$. pestis strains deficient in production of fulllength YopN or TyeA were grown under standard conditions $\left(37^{\circ} \mathrm{C}\right.$; with or without calcium), and the cell pellet and supernatant fractions were analyzed by SDS-PAGE and immunoblotting with $\alpha$-YopN and $\alpha$-TyeA antisera (Fig. 2B and C). $Y$. pestis KIM8-3002.P8 ( $\Delta$ tyeA) contains an in-frame deletion within tye $A$ eliminating the coding region for amino acid residues 10 to 55 of the 92-residue TyeA protein (8). Y. pestis KIM8-3002.P7 $(\Delta y o p N)$ carries an in-frame deletion within yop $N$ eliminating the coding sequence for residues 48 to 197 of the 293-residue YopN protein (19). The tye $A$ deletion strain expressed and secreted full-length YopN (32 kDa) and a ca. $37-\mathrm{kDa}$ protein that was recognized by both the $\alpha$-YopN and $\alpha$-TyeA antisera. The yop $N$ deletion strain expressed and secreted a ca. $26-\mathrm{kDa}$ protein that was recognized by the $\alpha$-TyeA antiserum. Thus, deletions within tye $A$ and yopN that disrupt the function of TyeA and YopN, respectively, each resulted in a corresponding reduction in the size of the $42-\mathrm{kDa} \alpha-Y o p N-$ and $\alpha$-TyeA-reactive band, indicating that this band represents a YopN-TyeA SDS-resistant complex or hybrid protein.

The $3^{\prime}$ end of the yop $N$ open reading frame overlaps the $5^{\prime}$ end of the tye $A$ open reading frame $(-2$ or +1 relative to the yop $N$ open reading frame) by 20 bp (Fig. 1), suggesting that a -2 or +1 translational frameshift event could produce a
YopN-TyeA hybrid protein. Translational frameshifts occur when the ribosome shifts its reading frame from the one it initiated translating to a new reading frame (11). A shift in the upstream direction is termed a -1 translational frameshift, whereas a shift in the downstream direction is termed a +1 translational frameshift. After a frameshift event has occurred, the ribosome will continue reading in the new frame until it encounters a termination codon. Programmed translational frameshift sites efficiently stimulate frameshifting within transcripts that require ribosomal frameshifts in order to produce a functional protein. In contrast, translational frameshift errors usually lead to a truncated, nonfunctional protein.

The YopN-TyeA hybrid protein is the product of a translational frameshift event. The yopN $-2 /+1$ open reading frame encodes a TGA stop codon +1 to yopN codon 271 (Fig. 1). A -2 or +1 frameshift prior to yop $N$ codon 271 would result in termination of translation prior to translation of the TyeA open reading frame. Thus, a translational frameshift event capable of producing the observed YopN-TyeA hybrid protein must occur after translation of yopN codon 270 and prior to termination of translation at the yopN termination codon.

A comparison of the $Y$. pestis KIM (24) and Y. enterocolitica 8081V (32) yopN sequences revealed the presence of a single nucleotide difference (Fig. 1) in the sequences preceding the 


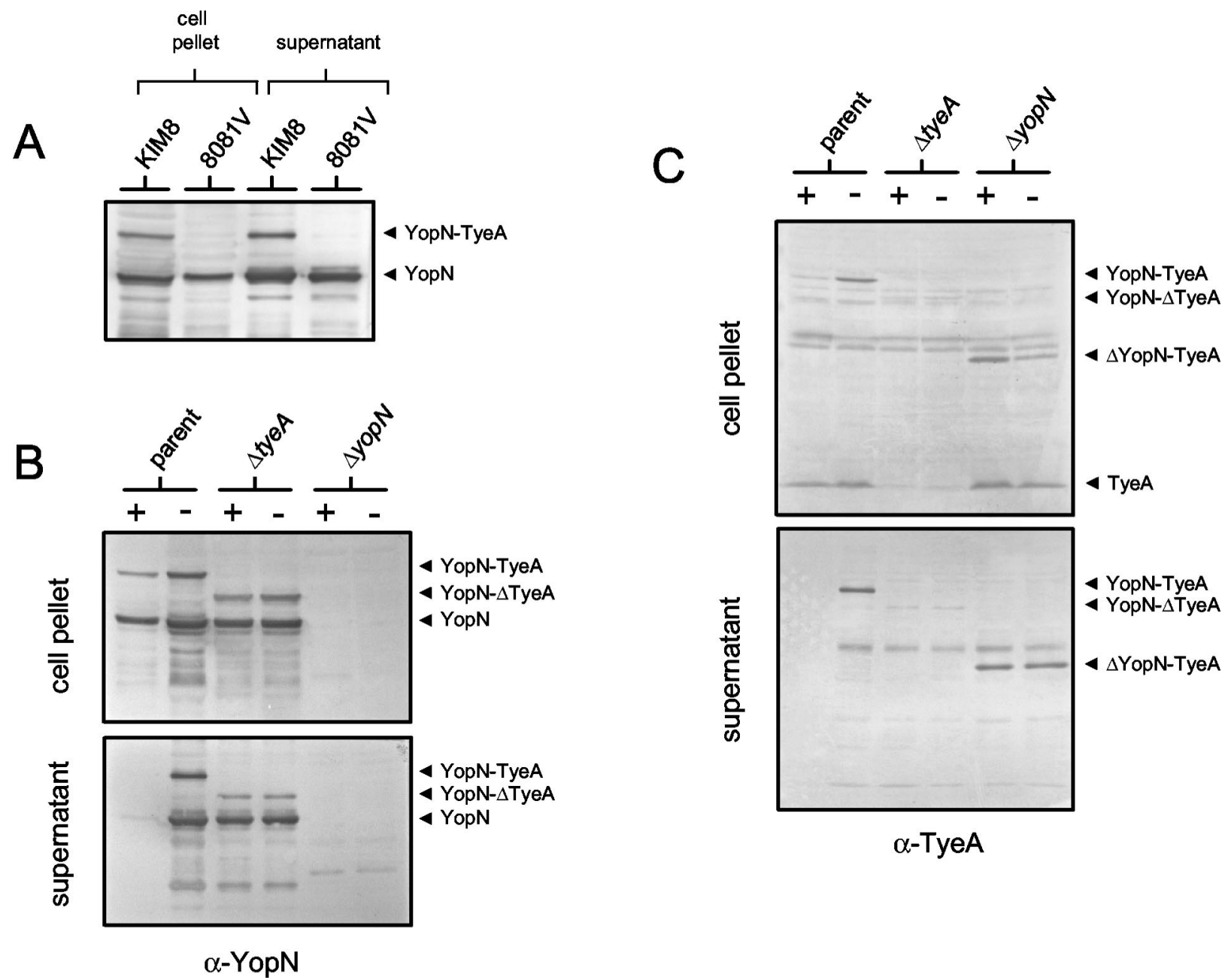

FIG. 2. Identification of a secreted YopN-TyeA hybrid protein. Bacterial strains were grown in TMH medium with $(+)$ or without $(-) 2.5 \mathrm{mM}$ $\mathrm{CaCl}_{2}$ for $5 \mathrm{~h}$ at $37^{\circ} \mathrm{C}$. Individual bacterial cultures were harvested by centrifugation and dissolved in SDS-PAGE sample buffer volumes corresponding to equal numbers of bacteria. Respective culture supernatants were precipitated with $10 \%$ trichloroacetic acid and resuspended in an equivalent volume of sample buffer. Samples were separated by SDS-PAGE, transferred to Immobilon-P membranes, and probed with antiserum specific for YopN (A and B) or TyeA (C). (A) Y. pestis KIM8 and Y. enterocolitica 8081V; (B and C) Y. pestis KIM8-3002 (parent), KIM8-3002.P8 ( $\Delta$ tyeA), and KIM8-3002.P7 ( $\Delta y o p N)$.

TyeA open reading frame. This A-to-T difference results in the placement of a TAA termination codon just upstream of the TyeA start codon in $Y$. enterocolitica. The presence of this termination codon would terminate translation of any $-2 /+1$ frameshift event that occurred prior to translation of yopN codon 287. The presence of this termination codon could explain why no YopN-TyeA hybrid protein is expressed by $Y$. enterocolitica and suggests that any translational frameshift event in $Y$. pestis and $Y$. pseudotuberculosis would likely occur after translation of yopN codon 270 and prior to translation of codon 287.

To determine if the YopN-TyeA hybrid protein is the result of a translational frameshift event, we examined the effect of several termination codons inserted within the yopN sequence on the production and size of the hybrid protein. To facilitate these studies, a $Y$. pestis $\Delta y o p N \Delta$ tye $A$ deletion strain was constructed using the PCR-ligation-PCR technique (1). The inframe deletion eliminated the coding sequence for amino acids 6 to 293 of YopN and 1 to 52 of TyeA. The DNA fragment containing the deletion was inserted into the suicide plasmid pRE112 (10) and moved into Y. pestis KIM8-3002 by allelic exchange as previously described (7), generating $Y$. pestis KIM8-3002.P55 ( $\Delta y o p N \Delta t y e A)$. Complementation of the deletion with plasmid $\mathrm{pYopN}$-TyeA restored expression of YopN and of the YopN-TyeA hybrid protein (see Fig. 5).

A series of pYopN-TyeA plasmids each containing a single nonsense mutation (termination codon) in the above identified region of yopN were constructed using the PCR-ligation-PCR technique (Fig. 1). The resultant plasmids were electroporated into the $Y$. pestis yopN tye $A$ deletion strain and assayed for production of the YopN-TyeA hybrid protein (Fig. 3). Insertion of a stop codon upstream of, or within, sequences required for a translational frameshift event would be expected to terminate translation at that point and eliminate production of the hybrid protein. Insertion of a stop codon downstream of the sequences required for a translational frameshift event would not be expected to affect the translational frameshift 

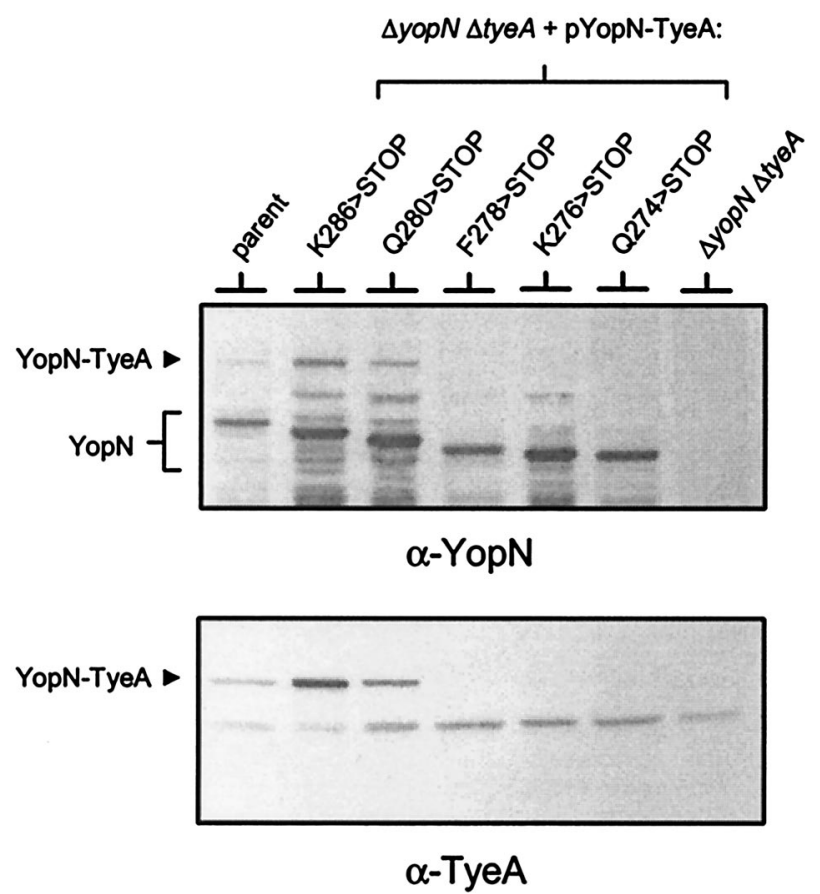

FIG. 3. Expression of the YopN-TyeA hybrid protein by $Y$. pestis KIM8-3002 (parent) and KIM8-3002.P55 ( $\Delta y o p N \Delta t y e A)$ carrying plasmid pYopN-TyeA (K286>STOP), pYopN-TyeA (Q280>STOP), pYopN-TyeA (F278 $>$ STOP), pYopN-TyeA (K276>STOP), or pYopNTyeA (Q274>STOP). Bacterial strains were grown in TMH medium lacking $\mathrm{CaCl}_{2}$ for $5 \mathrm{~h}$ at $37^{\circ} \mathrm{C}$. Individual bacterial cultures were harvested by centrifugation and dissolved in SDS-PAGE sample buffer volumes corresponding to equal numbers of bacteria. Samples were separated by SDS-PAGE, transferred to Immobilon-P membranes, and probed with antiserum specific for YopN $(\alpha-$ YopN) or TyeA $(\alpha$-TyeA).

process. However, sequences at some distance from a translational frameshift site can affect the efficiency of the translational frameshift process (11). Introduction of a termination codon at yopN codon 274 (Q274>STOP), 276 (K276>STOP), or 278 (F278>STOP) eliminated production of the YopNTyeA hybrid protein; in contrast, a nonsense mutation at codon 280 (Q280>STOP) or 286 (K286>STOP) did not affect YopN-TyeA hybrid protein production (Fig. 3). The estimated size of the truncated YopN protein produced by each of the nonsense mutants corresponded to the size predicted from the location of each termination codon; however, the sizes of the three YopN-TyeA hybrid proteins that were expressed failed to show a corresponding change in size. These data strongly suggest that the observed YopN-TyeA hybrid protein is the product of a translational frameshift event that occurs after translation of yop $N$ codon 270 and prior to translation of yopN codon 281. The termination codons inserted at yopN codons 274,276 , and 278 could prevent the translational frameshift event by terminating translation prior to encountering the frameshift site or by directly disrupting sequences required for the frameshift event per se.

Translational frameshift events are induced by distinct nucleotide sequences that cause the ribosome to pause over a specific sequence during translational elongation (11). The pause allows a kinetically unfavorable alternative coding event to occur. Scanning of the identified yopN nucleotide sequence for sequence motifs previously demonstrated to promote translational frameshift events revealed the presence of a UUUUGG dicodon that corresponds to the UUU-Ynn dicodon sequence motif that has been shown to be highly $(\sim 100$-fold more) frameshift prone in the rightward $(+1)$ direction $(14$, 31). A +1 frameshift at this site (Fig. 1) would generate a YopN-TyeA hybrid protein consisting of the first 278 residues of YopN, 9 residues (GNFFQRVKL) translated from the sequence upstream of the TyeA open reading frame and residues 1 to 92 of TyeA. The predicted YopN-TyeA hybrid protein would have a theoretical molecular weight of 42,713, which is nearly identical to the molecular weight estimated for the observed YopN-TyeA hybrid protein. In fact, a frameshift mutation engineered in yopN at the corresponding site (TTT-TGG; see below and Fig. 6) resulted in the expression of a YopNTyeA hybrid protein identical in size to that expressed via translational frameshifting, strongly suggesting that this is the site of the translational frameshift event. Numerous attempts to isolate enough of a truncated frameshifted YopN-TyeA protein in order to directly obtain the amino acid sequence at the potential translational frameshift site failed.

Interaction of TyeA with MBP-YopN and truncated MBPYopN proteins. The identified translational frameshift event results in the expression of a YopN-TyeA hybrid protein lacking the C-terminal 15 residues of YopN. To evaluate whether the truncated YopN protein might be capable of interacting with TyeA independently of the proposed translational frameshift event, we constructed a series of maltose binding protein (MBP)-fusion protein vectors that expressed either full-length MBP-YopN (MBP-YopN) or C-terminal-truncated MBPYopN proteins: MBP-YopN (R291>STOP), MBP-YopN (K286>STOP), MBP-YopN (Q280>STOP), MBP-YopN (F278>STOP), MBP-YopN (K276>STOP), MBP-YopN (Q274>STOP), and MBP-YopN (L265>STOP). TyeA was previously demonstrated to bind to YopN residues 242 to 293 (18), a region that includes a predicted coiled-coil region (residues 248 to 272). The ability of FLAG epitope-tagged TyeA to interact with the various truncated MBP-YopN proteins was evaluated via the protein affinity blotting (overlay) technique as previously described (9). The MBP-YopN proteins were separated by SDS-PAGE, transferred to Immobilon-P membranes, and probed with the cytosolic (soluble) fraction obtained from Escherichia coli BL21 carrying pFLAG-CTC-TyeA. FLAG-tagged TyeA bound strongly to full-length MBP-YopN, MBP-YopN (R291>STOP), and MBP-YopN (K286>STOP) (Fig. 4). MBP-YopN (Q280>STOP) and all further truncated versions of this hybrid protein failed to bind TyeA. These data indicate that the YopN-TyeA hybrid protein expressed by $Y$. pestis carrying pYopN-TyeA (Q280>STOP) (Fig. 3) is unlikely to be due to an SDS-resistant protein-protein interaction, since YopN (Q280>STOP) is not competent for interaction with TyeA by affinity blot analysis. These data also indicate that YopN truncations missing only the C-terminal 14 residues of YopN are unable to interact with TyeA, confirming that the extreme C-terminal region of YopN is required for this interaction.

Role of the YopN-TyeA hybrid protein in the regulation of Yop secretion. Translational frameshift events can be required for the production of a functional protein (programmed trans- 


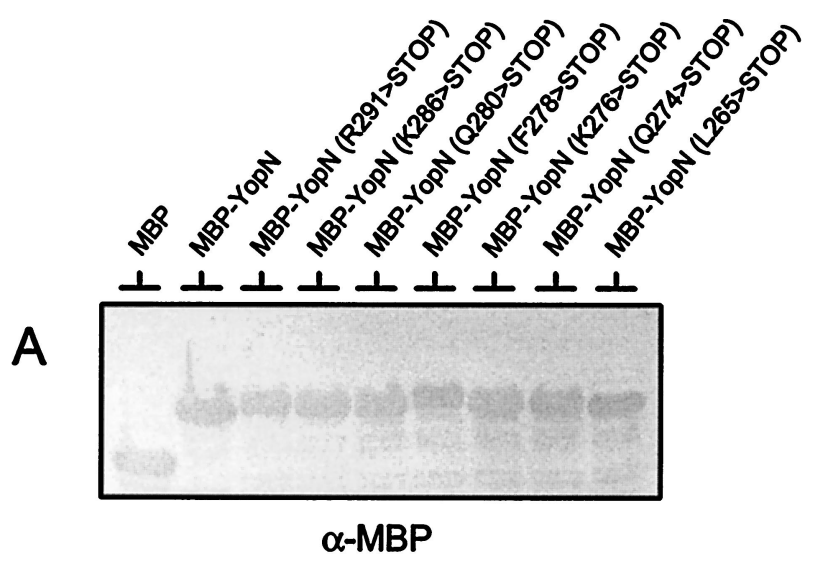

B
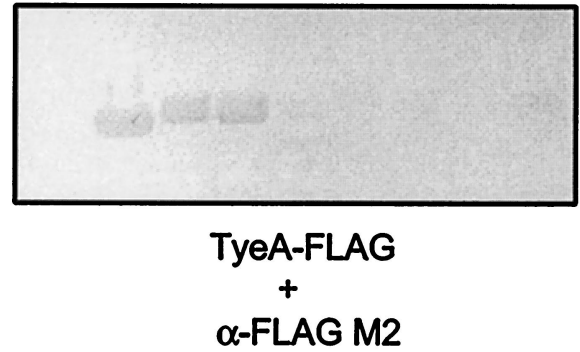

FIG. 4. Binding of TyeA-FLAG to MBP-YopN and C-terminaltruncated MBP-YopN proteins. Cell pellet fractions from E. coli BL21 cells expressing MBP, MBP-YopN, MBP-YopN (R291>STOP), MBP-YopN (K286>STOP), MBP-YopN (Q280>STOP), MBP-YopN (F278>STOP), MBP-YopN (K276>STOP), MBP-YopN (Q274> STOP), or MBP-YopN (L265>STOP) were separated by SDS-PAGE and transferred to Immobilon-P membranes. (A) Expression of MBP and MBP derivatives detected by immunoblot analysis with antiserum specific for MBP $(\alpha-\mathrm{MBP})$. (B) Duplicate Immobilon-P membrane probed with cytosolic extract from $E$. coli BL21 cells carrying plasmid pFLAG-CTC-TyeA. Bound TyeA-FLAG was detected with the FLAG M2 monoclonal antibody (Sigma).

lational frameshifts) or can result in the production of a truncated and/or nonfunctional protein (translational frameshift errors) (11). Interestingly, a database search revealed that several bacteria are predicted to exclusively express a secreted protein with an N-terminal domain homologous to YopN and a C-terminal domain homologous to TyeA (a YopN-TyeA-like hybrid protein). These proteins (CopN of Chlamydia spp. [12] and BopN of Bordetella spp. [36]) are also predicted to function in the regulation of TTS. These data suggest that a YopNTyeA hybrid protein could be functional. To begin to examine the functional significance of the YopN-TyeA hybrid protein in $Y$. pestis, we measured the calcium-regulated secretion of YopE, a translocated effector Yop, in strains expressing or not expressing the YopN-TyeA hybrid protein (Fig. 5).

The $Y$. pestis yopN tyeA deletion strain was transformed with plasmids expressing YopN (pYopN2 [7]), TyeA (pTyeA1 [8]), YopN and TyeA (pYopN2 and pTyeA1, and also YopN and TyeA with the YopN-TyeA hybrid protein [pYopN-TyeA]). By expressing YopN and TyeA from separate plasmids (pYopN2 and pTyeA1), we were able to express wild-type YopN and TyeA without expression of the YopN-TyeA hybrid protein. The parent strain ( $Y$. pestis KIM8-3002) and the yopN tyeA
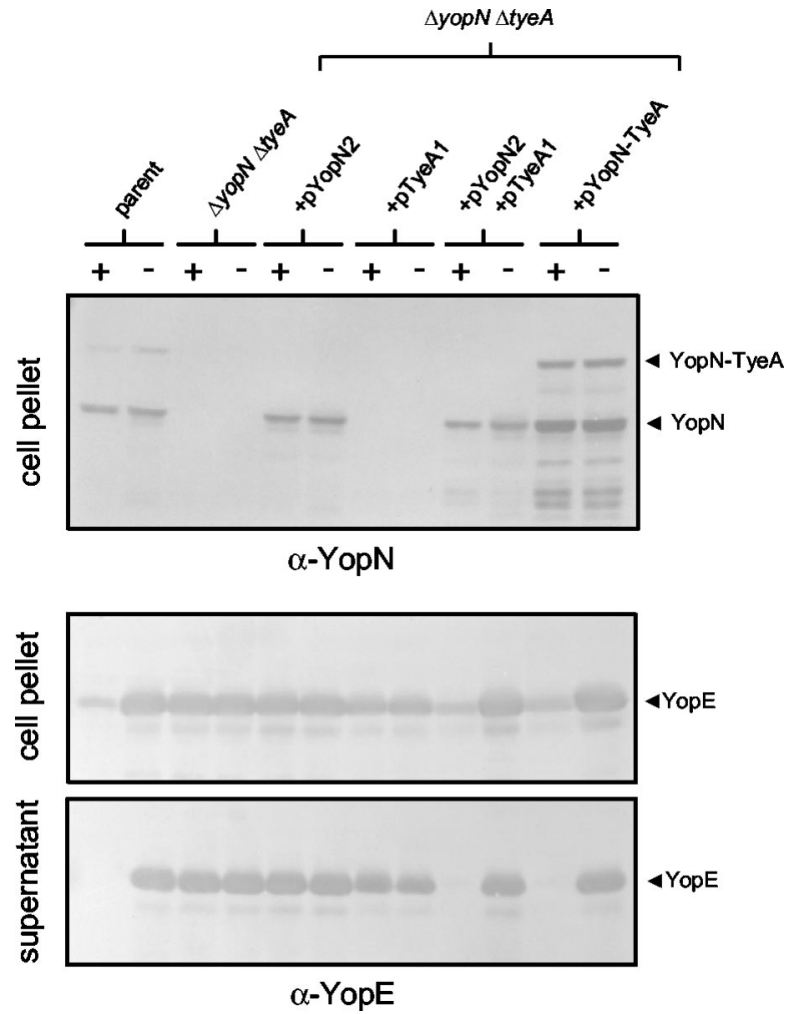

FIG. 5. Expression of the YopN-TyeA hybrid protein is not required for the calcium-dependent regulation of Yop secretion in $Y$. pestis. Y. pestis KIM8-3002 (parent) and KIM8-3002.P55 ( $\Delta y o p N$ $\Delta$ tyeA) carrying plasmids pYopN2, pTyeA1, pYopN2, and pTyeA1 or carrying pYopN-TyeA were grown in TMH medium with $(+)$ or without $(-) 2.5 \mathrm{mM} \mathrm{CaCl}$ for $5 \mathrm{~h}$ at $37^{\circ} \mathrm{C}$. Individual bacterial cultures were harvested by centrifugation and dissolved in SDS-PAGE sample buffer volumes corresponding to equal numbers of bacteria. Respective culture supernatants were precipitated with $10 \%$ trichloroacetic acid and resuspended in an equivalent volume of sample buffer. Samples were separated by SDS-PAGE, transferred to Immobilon-P membranes, and probed with antiserum specific for YopN $(\alpha-$ YopN $)$ or YopE $(\alpha-$ YopE$)$.

deletion strain carrying plasmid pYopN-TyeA both expressed the YopN-TyeA hybrid protein, whereas the other strains, as expected, did not express this protein (Fig. 5). As previously shown, failure to express either YopN or TyeA resulted in constitutive secretion of YopE in the presence and absence of calcium $(13,18)$. Complementation with either pYopN-TyeA or pYopN2 and pTyeA1 restored the calcium-dependent regulation of Yop secretion, indicating that the YopN-TyeA hybrid protein was not required for the calcium-dependent regulation of Yop secretion (Fig. 5). These data indicate that the translational frameshift event is not required for the regulation of Yop secretion in $Y$. pestis but do not rule on whether the YopN-TyeA hybrid is functional. These results are consistent with the fact that $Y$. enterocolitica shows calcium-regulated secretion but does not produce a YopN-TyeA hybrid protein.

To determine if the YopN-TyeA hybrid protein can function to regulate Yop secretion, we forced a +1 frameshift at the predicted site of the translational frameshift event by deleting a single $\mathrm{T}$ from the sequence TTT-TGG-C (new sequence, TTT-GGC) in plasmid pYopN-TyeA by using the PCR-liga- 

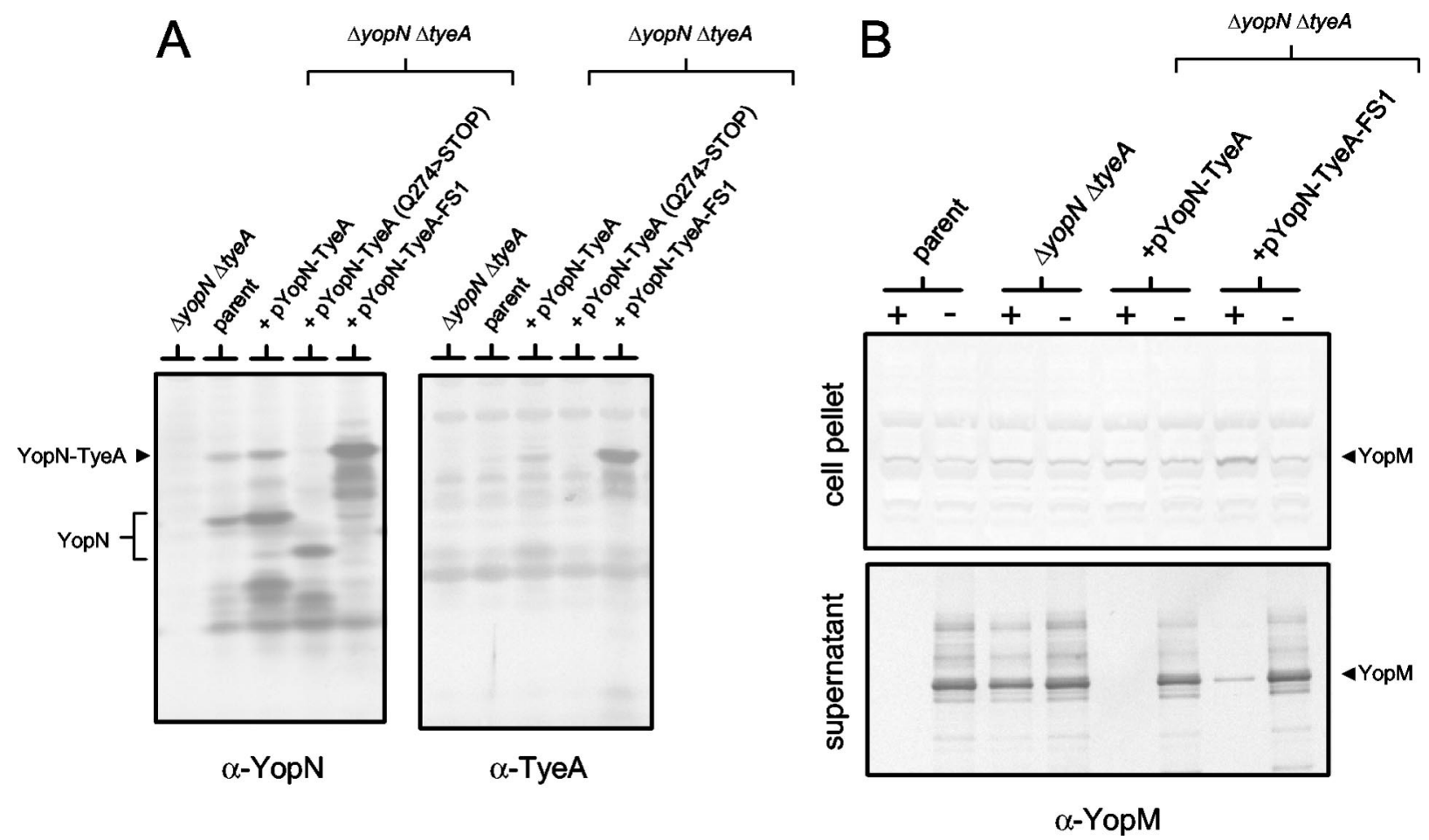

FIG. 6. Plasmid pYopN-TyeA-FS1 encodes a YopN-TyeA hybrid protein that restores calcium-dependent regulation of Yop secretion to $Y$. pestis KIM8-3002.P55 ( $\Delta y o p N \Delta$ tyeA). (A) Y. pestis KIM8-3002 (parent) and KIM8-3002.P55 ( $\Delta y o p N \Delta t y e A)$ carrying plasmids pYopN-TyeA, pYopN-TyeA (Q274>STOP), and pYopN-TyeA-FS1 were grown in TMH medium lacking $\mathrm{CaCl}_{2}$ for $5 \mathrm{~h}$ at $37^{\circ} \mathrm{C}$. Individual bacterial cultures were harvested by centrifugation and dissolved in SDS-PAGE sample buffer volumes corresponding to equal numbers of bacteria. Samples were separated by SDS-PAGE, transferred to Immobilon-P membranes, and probed with antiserum specific for YopN $(\alpha-Y o p N)$ or TyeA ( $\alpha$-TyeA). (B) Expression and secretion of YopM by Y. pestis KIM8-3002 (parent) and KIM8-3002.P55 ( $\Delta y o p N \Delta t y e A$ ) carrying plasmids pYopN-TyeA or pYopN-TyeA-FS1. Bacteria were grown in TMH medium with (+) or without (-) $2.5 \mathrm{mM} \mathrm{CaCl}_{2}$ for $5 \mathrm{~h}$ at $37^{\circ} \mathrm{C}$. Individual bacterial cultures were harvested by centrifugation and dissolved in SDS-PAGE sample buffer volumes corresponding to equal numbers of bacteria. Respective culture supernatants were precipitated with $10 \%$ trichloroacetic acid and resuspended in an equivalent volume of sample buffer. Samples were separated by SDS-PAGE, transferred to Immobilon-P membranes, and probed with antiserum specific for YopM $(\alpha-Y o p M)$.

tion-PCR technique (1) (Fig. 1). The resultant plasmid, termed pYopN-TyeA-FS1, was moved into the $Y$. pestis yopN tyeA deletion strain and analyzed for expression of YopN, TyeA, and the YopN-TyeA hybrid protein, as well as for secretion of YopM (Fig. 6). The yopN tyeA deletion strain carrying pYopNTyeA-FS1 expressed a YopN-TyeA hybrid protein of the same size as that expressed by the parent $Y$. pestis strain (Fig. 6A). No wild-type-size YopN was present in this strain; however, as expected, TyeA was expressed (data not shown). The yopN tyeA deletion strain expressing the frameshifted YopN-TyeA hybrid protein showed calcium-regulated YopM and YopE secretion (YopE data not shown), suggesting that the YopNTyeA hybrid protein was functional in regards to the regulation of Yop secretion (Fig. 6B). A small amount of YopM was secreted in the presence of calcium; however, the calciumdependent block in secretion associated with the frameshifted YopN-TyeA protein was nearly complete. It is unlikely that free TyeA binding to the YopN-TyeA hybrid protein is responsible for the calcium-dependent regulation of Yop secretion, since TyeA does not interact with either MBP-YopN (F278 $>$ STOP) or MBP-YopN (Q280 $>$ STOP) (Fig. 4). Further, plasmids pYopN-TyeA (F278 $>$ STOP) and pYopN-TyeA $(\mathrm{Q} 280>$ STOP) were unable to restore calcium-dependent regulation of secretion to either a $Y$. pestis yopN or yopN tyeA deletion strain (data not shown), indicating that YopN truncated following translation of yopN codons 277 or 279 is not functional unless directly fused to TyeA by a frameshift mutation or a translational frameshift event. These data suggest that the translational frameshift event that generates the secreted YopN-TyeA hybrid protein results in the production of a functional YopN-TyeA hybrid protein that can be secreted via the TTS system. In addition to a possible role in the regulation of Yop secretion, the YopN-TyeA hybrid protein could have a not-yet-identified role in TTS, translocation, or virulence.

The results presented confirm that $Y$. pestis expresses and secretes a functional YopN-TyeA hybrid protein that is the product of a translational frameshift event. The identification of this hybrid protein explains the presence of the unique ca. 42-kDa band observed in culture supernatants isolated from $Y$. pestis and $Y$. pseudotuberculosis, but not in those from $Y$. enterocolitica. The expression and secretion of this hybrid protein may have led to some of the initial conflicting results on whether TyeA was cytosolic or cell surface associated $(5,18)$. Our results support the current hypothesis that TyeA is a cytosolic protein that directly interacts with YopN and remains cytosolic following secretion of YopN.

The identification of a functional secreted YopN-TyeA hybrid protein in $Y$. pestis suggests that the reversible binding of 
TyeA to YopN per se is not the key event controlling the opening and closing of the secretion channel. Although both TyeA and YopN are essential for the regulation of Yop secretion, it appears that they can function if expressed independently or as a single hybrid protein. These findings also suggest that the YopN-TyeA-like hybrid proteins expressed by Chlamydia spp. (CopN) and Bordetella spp. (BopN) are also likely to function in the regulation of TTS. At this point it is unclear why these bacteria express a YopN-TyeA-like hybrid protein instead of two separate interacting proteins. As more is learned about the Chlamydia spp. and Bordetella spp. TTS systems and the signals that control secretion by these systems, light may be shed on the advantages and/or disadvantages of expressing these key regulatory proteins as a single protein.

This work was supported by Public Health Service grants AI 39575 and AI 50552 from the National Institutes of Health to G.P.

\section{REFERENCES}

1. Ali, S. A., and A. Steinkasserer. 1995. PCR-ligation-PCR mutagenesis: a protocol for creating gene fusions and mutations. BioTechniques 18:746750.

2. Ben-Gurion, R., and A. Shafferman. 1981. Essential virulence determinants of different Yersinia species are carried on a common plasmid. Plasmid 5:183-187.

3. Bolin, I., L. Norlander, and H. Wolf-Watz. 1982. Temperature-inducible outer membrane protein of Yersinia pseudotuberculosis and Yersinia enterocolitica is associated with the virulence plasmid. Infect. Immun. 37:506-512.

4. Cheng, L. W., O. Kay, and O. Schneewind. 2001. Regulated secretion of YopN by the type III machinery of Yersinia enterocolitica. J. Bacteriol. 183: 5293-5301.

5. Cheng, L. W., and O. Schneewind. 2000. Yersinia enterocolitica TyeA, an intracellular regulator of the type III machinery, is required for specific targeting of YopE, YopH, YopM, and YopN into the cytosol of eukaryotic cells. J. Bacteriol. 182:3183-3190.

6. Cornelis, G. R. 2000. Molecular and cell biology aspects of plague. Proc. Natl. Acad. Sci. USA 97:8778-8783.

7. Day, J. B., F. Ferracci, and G. V. Plano. 2003. Translocation of YopE and YopN into eukaryotic cells by Yersinia pestis yopN, tye A, sycN, yscB and $l c r G$ deletion mutants measured using a phosphorylatable peptide tag and phosphospecific antibodies. Mol. Microbiol. 47:807-823.

8. Day, J. B., and G. V. Plano. 1998. A complex composed of SycN and YscB functions as a specific chaperone for YopN in Yersinia pestis. Mol. Microbiol 30:777-788.

9. Day, J. B., and G. V. Plano. 2000. The Yersinia pestis YscY protein directly binds YscX, a secreted component of the type III secretion machinery. J. Bacteriol. 182:1834-1843.

10. Edwards, R. A., L. H. Keller, and D. M. Schifferli. 1998. Improved allelic exchange vectors and their use to analyze 987P fimbria gene expression. Gene 207:149-157.

11. Farabaugh, P. J. 1996. Programmed translational frameshifting. Annu. Rev. Genet. 30:507-528.

12. Fields, K. A., and T. Hackstadt. 2000. Evidence for the secretion of Chlamydia trachomatis CopN by a type III secretion mechanism. Mol. Microbiol. 38:1048-1060.

13. Forsberg, A., A. M. Viitanen, M. Skurnik, and H. Wolf-Watz. 1991. The surface-located YopN protein is involved in calcium signal transduction in Yersinia pseudotuberculosis. Mol. Microbiol. 5:977-986.

14. Fu, C., and J. Parker. 1994. A ribosomal frameshifting error during trans lation of the $\arg I$ mRNA of Escherichia coli. Mol. Gen. Genet. 243:434-441.

15. Grosdent, N., I. Maridonneau-Parini, M. P. Sory, and G. R. Cornelis. 2002
Role of Yops and adhesins in resistance of Yersinia enterocolitica to phagocytosis. Infect. Immun. 70:4165-4176.

16. Hueck, C. J. 1998. Type III protein secretion systems in bacterial pathogens of animals and plants. Microbiol. Mol. Biol. Rev. 62:379-433.

17. Iriarte, M., and G. R. Cornelis. 1999. Identification of SycN, YscX, and YscY, three new elements of the Yersinia yop virulon. J. Bacteriol. 181:675680

18. Iriarte, M., M. P. Sory, A. Boland, A. P. Boyd, S. D. Mills, I. Lambermont, and G. R. Cornelis. 1998. TyeA, a protein involved in control of Yop release and in translocation of Yersinia Yop effectors. EMBO J. 17:1907-1918.

19. Jackson, M. W., J. B. Day, and G. V. Plano. 1998. YscB of Yersinia pestis functions as a specific chaperone for YopN. J. Bacteriol. 180:4912-4921.

20. Matson, J. S., and M. L. Nilles. 2001. LcrG-LcrV interaction is required for control of Yops secretion in Yersinia pestis. J. Bacteriol. 183:5082-5091.

21. Michiels, T., P. Wattiau, R. Brasseur, J. M. Ruysschaert, and G. Cornelis. 1990. Secretion of Yop proteins by yersiniae. Infect. Immun. 58:2840-2849.

22. Orth, K., L. E. Palmer, Z. Q. Bao, S. Stewart, A. E. Rudolph, J. B. Bliska, and J. E. Dixon. 1999. Inhibition of the mitogen-activated protein kinase kinase superfamily by a Yersinia effector. Science 285:1920-1923.

23. Palmer, L. E., S. Hobbie, J. E. Galan, and J. B. Bliska. 1998. YopJ of Yersinia pseudotuberculosis is required for the inhibition of macrophage TNF-alpha production and downregulation of the MAP kinases p38 and JNK. Mol. Microbiol. 27:953-965.

24. Perry, R. D., S. C. Straley, J. D. Fetherston, D. J. Rose, J. Gregor, and F. R. Blattner. 1998. DNA sequencing and analysis of the low- $\mathrm{Ca}^{2+}$-response plasmid pCD1 of Yersinia pestis KIM5. Infect. Immun. 66:4611-4623.

25. Persson, C., R. Nordfelth, A. Holmstrom, S. Hakansson, R. Rosqvist, and H. Wolf-Watz. 1995. Cell-surface-bound Yersinia translocate the protein tyrosine phosphatase YopH by a polarized mechanism into the target cell. Mol. Microbiol. 18:135-150.

26. Portnoy, D. A., H. Wolf-Watz, I. Bolin, A. B. Beeder, and S. Falkow. 1984 Characterization of common virulence plasmids in Yersinia species and their role in the expression of outer membrane proteins. Infect. Immun. 43:108114.

27. Rosqvist, R., A. Forsberg, and H. Wolf-Watz. 1991. Intracellular targeting of the Yersinia YopE cytotoxin in mammalian cells induces actin microfilament disruption. Infect. Immun. 59:4562-4569.

28. Rosqvist, R., K. E. Magnusson, and H. Wolf-Watz. 1994. Target cell contact triggers expression and polarized transfer of Yersinia YopE cytotoxin into mammalian cells. EMBO J. 13:964-972.

29. Russmann, H., U. Gerdemann, E. I. Igwe, K. Panthel, J. Heesemann, S. Garbom, H. Wolf-Watz, and G. Geginat. 2003. Attenuated Yersinia pseudotuberculosis carrier vaccine for simultaneous antigen-specific CD4 and CD8 T-cell induction. Infect. Immun. 71:3463-3472.

30. Schesser, K., A. K. Spiik, J. M. Dukuzumuremyi, M. F. Neurath, S. Pettersson, and H. Wolf-Watz. 1998. The yopJ locus is required for Yersinia-mediated inhibition of NF-кB activation and cytokine expression: YopJ contains a eukaryotic SH2-like domain that is essential for its repressive activity. Mol. Microbiol. 28:1067-1079.

31. Schwartz, R., and J. F. Curran. 1997. Analyses of frameshifting at UUUpyrimidine sites. Nucleic Acids Res. 25:2005-2011.

32. Snellings, N. J., M. Popek, and L. E. Lindler. 2001. Complete DNA sequence of Yersinia enterocolitica serotype O:8 low-calcium-response plasmid reveals a new virulence plasmid-associated replicon. Infect. Immun. 69:46274638.

33. Straley, S. C., G. V. Plano, E. Skrzypek, P. L. Haddix, and K. A. Fields. 1993 Regulation by $\mathrm{Ca}^{2+}$ in the Yersinia low- $\mathrm{Ca}^{2+}$ response. Mol. Microbiol. 8:1005-1010.

34. Williams, A. W., and S. C. Straley. 1998. YopD of Yersinia pestis plays a role in negative regulation of the low-calcium response in addition to its role in translocation of Yops. J. Bacteriol. 180:350-358.

35. Yother, J., and J. D. Goguen. 1985. Isolation and characterization of $\mathrm{Ca}^{2+}$ blind mutants of Yersinia pestis. J. Bacteriol. 164:704-711.

36. Yuk, M. H., E. T. Harvill, P. A. Cotter, and J. F. Miller. 2000. Modulation of host immune responses, induction of apoptosis and inhibition of NF-кB activation by the Bordetella type III secretion system. Mol. Microbiol. 35: 991-1004. 